\title{
Asymptomatic Gastric Band Erosion Detected during Routine Gastroduodenoscopy
}

\author{
Gee Young Yun, Woo Sub Kim, Hye Jin Kim, Sun Hyung Kang, Hee Seok Moon, Jae Kyu Sung and Hyun Yong Jeong \\ Department of Internal Medicine, Chungnam National University School of Medicine, Daejeon, Korea
}

The incidence of gastric band erosion has decreased to $1 \%$. Gastric band erosion can manifest with various clinical symptoms, although some patients remain asymptomatic. We present a case of a mostly asymptomatic patient who was diagnosed with gastric band erosion during a routine health check-up. A 32-year-old man without any underlying diseases except for non-alcoholic fatty liver underwent laparoscopic adjustable gastric band surgery in 2010. He had no significant complications postoperatively. He underwent routine health check-ups with near-normal gastroduodenoscopic findings through 2014. However, in 2015, routine gastroduodenoscopy showed that the gastric band had eroded into the stomach. His gastric band was removed laparoscopically, and the remaining gastric ulcer perforation was repaired using an omental patch. Due to the early diagnosis, the infection was not serious. The patient was discharged on postoperative day 3 with oral antibiotics. This patient was fortunately diagnosed early by virtue of a routine health check-up; thus, eliminating the possibility of serious complications. Clin Endosc 2016;49:294-297

Key Words: Laparoscopic gastric band; Band erosion; Obesity, morbid; Bariatric surgery

\section{INTRODUCTION}

Obesity has turned into one of the most crucial public health issues worldwide; thus, bariatric surgery has correspondingly become more popular worldwide. ${ }^{1}$ A recent study reported that bariatric surgery has an excellent effect on weight loss in comparison with conventional medical treatment in extremely obese Korean patients, and improvement of comorbidities was also better. ${ }^{2}$ Laparoscopic adjustable gastric band (LAGB) surgery, laparoscopic Roux-en-Y gastric bypass, and laparoscopic sleeve gastrectomy are the three most common bariatric procedures performed in Korea. Bariatric surgery, in the form of a laparoscopic sleeve gastrectomy procedure, was first performed in this country in January $2003 .{ }^{3}$

Received: November 29, 2015 Revised: January 3, 2016

Accepted: January 4, 2016

Correspondence: Hee Seok Moon

Department of Internal Medicine, Chungnam National University School of Medicine, 266 Munhwa-ro, Jung-gu, Daejeon 35015, Korea

Tel: +82-42-280-7143, Fax: +82-42-257-5753, E-mail: mhs1357@cnu.ac.kr

(cc) This is an Open Access article distributed under the terms of the Creative Commons Attribution Non-Commercial License (http://creativecommons.org/ licenses/by-nc/3.0) which permits unrestricted non-commercial use, distribution, and reproduction in any medium, provided the original work is properly cited.
Subsequently, a laparoscopic gastric bypass was performed in the same year. In August 2004, the first LAGB was performed. ${ }^{4,5}$

LAGB is the most favored bariatric surgery in most countries, including Korea. ${ }^{6}$ LAGB has been proven to be a safe operation with a 7 to 10 times lower mortality rate than bypass surgery of the stomach. In addition, it has few perioperative complications. However, late complications of LAGB, such as band slippage, gastric prolapse, gastric necrosis, band erosion, pouch dilatation, and esophageal reflux, do occur and must be dealt with cautiously. ${ }^{7}$

The prevalence of gastric band erosion was initially reported to be $10 \%$, but because of improvements in the technique, it has decreased to $1 \%{ }^{2}$ Gastric band erosion can manifest with various clinical symptoms, such as abdominal pain, obstruction, turbid fluid, and recurrent port infections, although some patients remain asymptomatic. Some seemingly insignificant symptoms, such as loss of restriction or weight gain, may also indicate gastric band erosion. ${ }^{6}$ We present a case of a mostly asymptomatic patient who was diagnosed early with gastric band erosion during a routine health check-up. 


\section{CASE REPORT}

A 32-year-old man without any underlying diseases except for non-alcoholic fatty liver underwent LAGB surgery in 2010. Prior to surgery, his weight had been $133 \mathrm{~kg}$. With a height of $180 \mathrm{~cm}$, his body mass index (BMI) was calculated as $41.8 \mathrm{~kg} /$ $\mathrm{m}^{2}$. The indication for bariatric surgery in Asian patients is a $\mathrm{BMI}>35 \mathrm{~kg} / \mathrm{m}^{2}$ or a $\mathrm{BMI}>30 \mathrm{~kg} / \mathrm{m}^{2}$ with diabetes or two other obesity-related comorbidities. ${ }^{5}$ Thus, he was a good candidate for bariatric surgery. He had no significant complications postoperatively, and his weight gradually decreased to $97.4 \mathrm{~kg}$, reflecting a concomitant decrease in BMI to 29.8 $\mathrm{kg} / \mathrm{m}^{2}$. The patient was satisfied with the surgery. He had undergone routine health check-ups at Chungnam National University Hospital in 2012, 2014, and 2015. At each checkup, he underwent gastroduodenoscopy and colon fiberscopy, with near-normal gastroduodenoscopic findings through 2014 (Fig. 1); prior to 2015, his only symptom was mild reflux esophagitis resulting in heartburn. There was also no ev- idence of gastric band erosion or any other complications although he had regained $5 \mathrm{~kg}$ over the course of the previous year. However, in 2015 routine gastroduodenoscopy showed that the gastric band had eroded into the stomach (Fig. 1). Abdominal and pelvic computed tomography performed in 2015 showed that the gastric band eroding into the stomach had causing a perforation (Fig. 2). We immediately sent him to the hospital where he had originally undergone LAGB surgery. His gastric band was removed laparoscopically, and the remaining gastric ulcer perforation was repaired using an omental patch. Due to the early diagnosis, the infection was not serious. There was only a small amount of exudate around the gastric ulcer and localized peritonitis. The patient was discharged on postoperative day 3 with oral antibiotics.

\section{DISCUSSION}

The most frequently used bariatric procedure in South
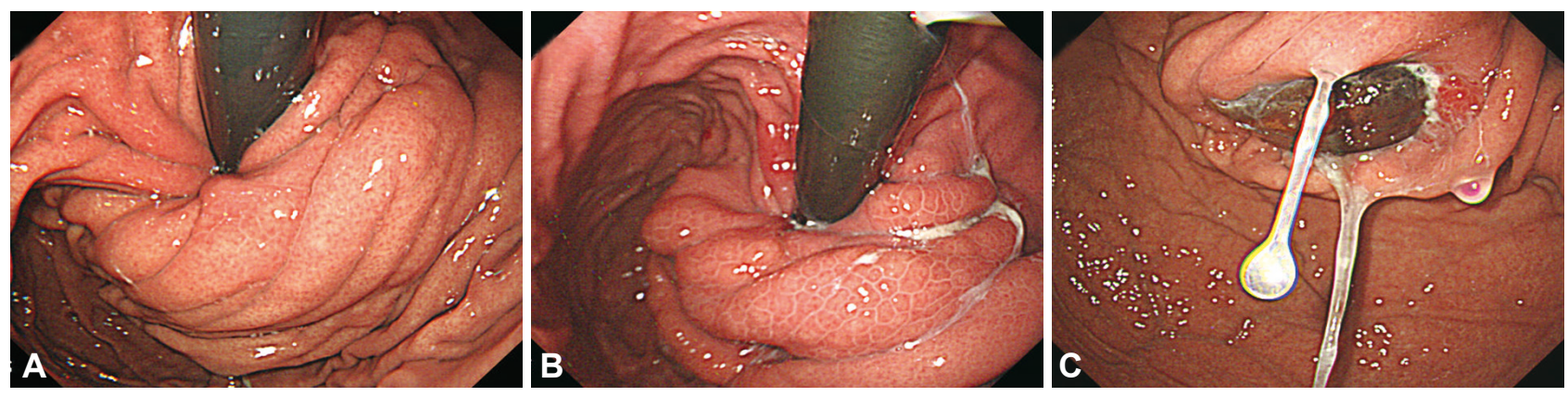

Fig. 1. Endoscopic finding in the fundus area during routine gastroduodenoscopy. (A) Three years ago, routine gastroduodenoscopy shows an intact gastric mucosa. (B) One year ago, routine gastroduodenoscopy shows an intact gastric mucosa. (C) Recently, routine gastroduodenoscopy shows that the gastric band had eroded into the stomach with gastric ulcer perforation.
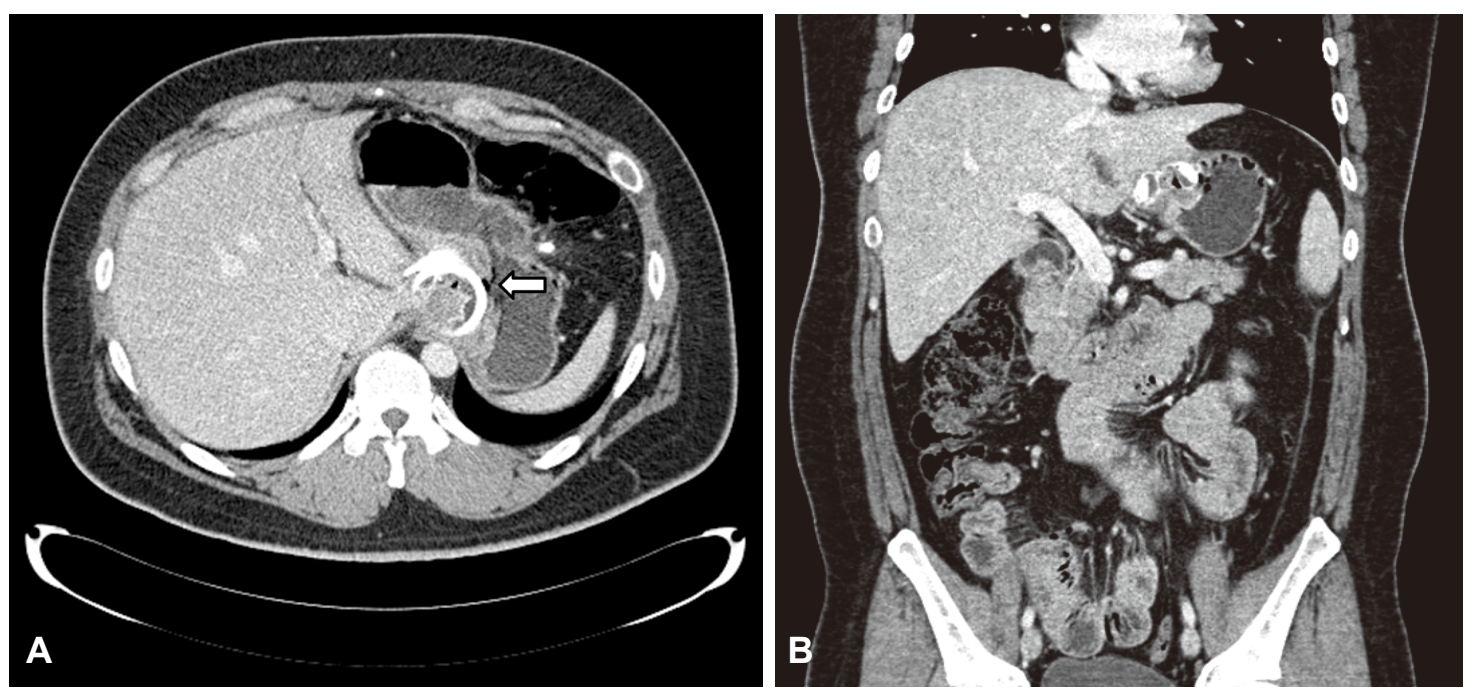

Fig. 2. Abdominal and pelvic computed tomography for a routine health-check up in 2015. (A) Axial view, the gastric band partially eroded into the gastric lumen. The arrow shows that the gastric band caused gastric perforation. (B) Sagittal view, the eroded band is shown in the gastric lumen with gastric perforation. 
Korea is LAGB, which comprises approximately $70 \%$ of all national bariatric procedures. ${ }^{4}$ The reasons for its popularity include reversibility, adjustability, simplicity, and a relatively low rate of perioperative complications. ${ }^{8}$

The current prevalence of band erosion after LAGB reported in published series and large-scale studies ranges from $0.6 \%$ to $2.2 \%{ }^{6,9-14}$ In one study, the timing of diagnosis of band erosion ranged from months to years (6 to 132 months) after the initial surgery. ${ }^{6}$

Occasionally, patients with band erosion are asymptomatic. Symptoms of band erosion include recurrent port infections, abdominal pain, bowel obstruction, and rarely, sepsis with peritonitis. The presentations are not usually serious and perforation of the intraperitoneal lumen is rare, which may be due to adhesion development prior to complete perforation of the gastric wall. Nonetheless, some patients become acutely ill with bowel obstruction or sepsis with peritonitis, which warrants immediate intervention. To diagnose these complications, it is important to be aware of them. Some patients who have done well in controlling their body weight may suddenly struggle with controlling their food intake even if the gastric band fluid volume is adequate. . $7,75-18^{-18}$

The etiology of band erosion is not fully understood. Early erosions may be secondary to early infections or undiagnosed gastric perforations that occur during the LAGB procedure. Late erosions may be caused by chronic ischemia of the gastric wall due to the high pressure exerted by the gastric band. Port site infection is one of the causes of gastric band erosion due to the migration of bacteria from the stomach. ${ }^{6,16,19}$

As mentioned previously, our patient had a late erosion, diagnosed 5 years after LAGB. We suspected that the etiology of his band erosion was chronic ischemia. Previous studies have not specified the proportion of asymptomatic patients with late erosions. However, it is difficult to diagnose band erosion when the symptoms are not clear, but a loss of weight restriction and an increasing appetite are important clues in diagnosing gastric band erosions. Surgeons should remove the gastric band after diagnosing gastric band erosion, and it is important to diagnose it early to prevent further complications. Routine gastroduodenoscopy may be indispensable for detecting asymptomatic patients with gastric band erosion. No previous studies have reported the use of gastroduodenoscopy after LAGB, and there is no suggested interval for routine gastroduodenoscopy after LAGB. Thus, clinicians should also think about the cost-effectiveness of performing routine gastroduodenoscopy after LAGB.

Our case presents a mostly asymptomatic patient with gastric band erosion. The only symptoms he experienced were heartburn 1 month previously, which can be explained by reflux esophagitis, and weight regain of about $5 \mathrm{~kg}$ during the previous year, which was not deemed significant.

Gastroduodenoscopy is the gold standard diagnostic tool for gastric band erosion. Our patient was fortunately diagnosed early by virtue of a routine health check-up, thus eliminating the possibility of serious complications. In Korea, upper esophagogastroduodenoscopy (EGD) is recommended for people over 40 every 2 years to screen for gastric cancer. Although annual upper EGD is not necessary for patients after LAGB, symptoms such as loss of restriction, weight regain, and increased appetite may indicate the need for EGD.

\section{Conflicts of Interest}

The authors have no financial conflicts of interest.

\section{REFERENCES}

1. Park JY, Song D, Kim YJ. Causes and outcomes of revisional bariatric surgery: initial experience at a single center. Ann Surg Treat Res 2014;86:295-301.

2. Heo YS, Park JM, Kim YJ, et al. Bariatric surgery versus conventional therapy in obese Korea patients: a multicenter retrospective cohort study. J Korean Surg Soc 2012;83:335-342.

3. Han SM, Kim WW. The 3-year results of laparoscopic sleeve gastrectomy for the treatment of Korean morbid obesity. J Korean Surg Soc 2007;73:400-405.

4. Lee SK. Current status of laparoscopic metabolic/bariatric surgery in Korea. J Minim Invasive Surg 2015;18:59-62.

5. Choi SH. Bariatric surgery. Historical review and perspective. J Metab Bariatr Surg 2012;1:1-5.

6. Chisholm J, Kitan N, Toouli J, Kow L. Gastric band erosion in 63 cases: endoscopic removal and rebanding evaluated. Obes Surg 2011;21:16761681.

7. Lee H. Complications of surgery for morbid obesity: laparoscopic adjustable gastric banding. J Metab Bariatr Surg 2012;1:67-72.

8. Park YC. Laparoscopic adjustable gastric banding. J Metab Bariatr Surg 2012;1:30-37.

9. Favretti F, Segato G, Ashton D, et al. Laparoscopic adjustable gastric banding in 1,791 consecutive obese patients: 12-year results. Obes Surg 2007;17:168-175.

10. Regusci L, Groebli Y, Meyer JL, Walder J, Margalith D, Schneider R. Gastroscopic removal of an adjustable gastric band after partial intragastric migration. Obes Surg 2003;13:281-284.

11. Ponce J, Lindsey B, Pritchett S, Bleech M, Marlowe K. New adjustable gastric bands available in the United States: a comparative study. Surg Obes Relat Dis 2011;7:74-79.

12. O'Brien PE, Dixon JB. Weight loss and early and late complications: the international experience. Am J Surg 2002;184(6B):42S-45S.

13. Vella M, Galloway DJ. Laparoscopic adjustable gastric banding for severe obesity. Obes Surg 2003;13:642-648.

14. Niville E, Dams A, Vlasselaers J. Lap-Band erosion: incidence and treatment. Obes Surg 2001;11:744-747.

15. Busetto L, Segato G, De Marchi F, et al. Outcome predictors in morbidly obese recipients of an adjustable gastric band. Obes Surg 2002;12:83-92.

16. Abu-Abeid S, Keidar A, Gavert N, Blanc A, Szold A. The clinical spectrum of band erosion following laparoscopic adjustable silicone gastric banding for morbid obesity. Surg Endosc 2003;17:861-863. 
17. Angrisani L, Alkilani M, Basso N, et al. Laparoscopic Italian experience with the Lap-Band. Obes Surg 2001;11:307-310.

18. Silecchia G, Restuccia A, Elmore U, et al. Laparoscopic adjustable silicone gastric banding: prospective evaluation of intragastric migration of the lap-band. Surg Laparosc Endosc Percutan Tech 2001;11:229-234.

19. Nehoda H, Weiss H, Labeck B, et al. Results and complications after adjustable gastric banding in a series of 250 patients. Am J Surg 2001;181:12-15. 